\title{
Influence of supragingival biofilm control and smoking habit on Interleukin-1 $\beta$ concentration
}

\section{Sabrina Carvalho GOMES(a) Carolina Coradini ABASCAL(b) Alex Nogueira HAAS(a) Patricia Daniela Melchiors ANGST(c) Rui Vicente OPPERMANN(a) Rosemary Adriana Chierici MARCANTONIO(d)}

(a) Universidade Federal do Rio Grande do Sul - UFRGS, Dental School, Department of Periodontology, Porto Alegre, RS, Brazil.

(b) Universidade Luterana do Brasil - ULBRA, Dental School, Post Graduate Program, Canoas, RS, Brazil.

(c) Universidade Federal do Rio Grande do Sul - UFRGS, Dental School, Post Graduate Program, Porto Alegre, RS, Brazil.

(d) Universidade Estadual Paulista - UNESP, Dental School, Department of Diagnoses and Surgery, Araraquara, SP, Brazil.

Declaration of Interests: The authors certify that they have no commercial or associative interest that represents a conflict of interest in connection with the manuscript.

Corresponding Author:

Sabrina Carvalho Gomes

E-mail: sabrinagomes.perio@gmail.com

DOI: 10.1590/1807-3107BOR-2015.vol29.0115

Submitted: Jan 23, 2015

Accepted for publication: Jun 12, 2015

Last revision: Aug 27, 2015

\begin{abstract}
This investigation compared gingival crevicular fluid (GCF) interleukin-1 $\beta$ (IL-1 $\beta)$ concentrations in periodontitis patients subjected to a strict supragingival biofilm control (Supra) for 6 months. Never-smokers (23) and smokers ( $n=20 ; 19.6 \pm 11.8$ cigarettes/day) moderate-to-severe chronic periodontitis patients underwent a 6 months period of supragingival control with weekly recall visits. Periodontal probing depth (PPD), bleeding on probing (BOP) and GCF samples (from different PPD category sites: 3-5 mm and $6-10 \mathrm{~mm}$ ) were obtained at the baseline, 30, and 180 days. IL-1 $\beta$ was assessed by enzyme-linked immunosorbent assay. Generalized estimating equations were used to fit prediction models of IL-1 $\beta$ changes, considering the dependence between the examinations, and using only data from experimental sites. Overall IL-1 $\beta$ concentrations decreased from $3.2 \mathrm{pg} / \mu \mathrm{L}$ to $1.9 \mathrm{pg} / \mu \mathrm{L}$. Higher baseline IL-1 $\beta$ concentrations were associated with higher baseline PPD values in both groups. There were no differences in IL-1 $\beta$ concentrations between never-smokers and smokers over time for any PPD category. Higher baseline PPD values and the presence of BOP on day 180 were significantly associated with higher IL-1 $\beta$ concentrations. A strict Supra regimen reduced IL-1 $\beta$ concentrations over time in periodontitis patients. The benefits observed for smokers underline the importance of oral hygiene measures, even considering the presence of this important risk factor.
\end{abstract}

Keywords: Dental Plaque; Interleukin-1Beta; Periodontal Disease; Smoking.

\section{Introduction}

Interleukin $1 \beta$ (IL-1 $\beta$ ) in gingival crevicular fluid (GCF) is an important subgingival biomarker of inflammatory status. Studies have shown an increase in its levels associated with periodontal disease severity and a decrease after periodontal therapy., ${ }^{1,2}$ There is evidence of IL-1 $\beta$ influence in the modulation of bone resorption; stimulation of the production of osteoclasts, proteases, prostaglandin-E2, and metalloproteinases; and synthesis of collagenase. ${ }^{3}$ This cytokine is associated with pro-inflammatory behavior, meaning that IL-1 $\beta$ helps to trigger and promote periodontal tissue damage in response to bacterial challenge. ${ }^{4}$

Contributing to periodontal destruction, tobacco consumption, a well-known risk factor in periodontitis, ${ }^{5}$ impairs the immune system, ${ }^{6}$ 
inhibiting neutrophil and macrophage action and affecting defense against bacteria. ${ }^{6}$ Smoking also reduces the proliferative capacity of $\mathrm{T}$ and $\mathrm{B}$ cells and the production of antibodies, ${ }^{7}$ in turn inhibiting host response to periodontal therapy. ${ }^{8,9}$

Supragingival biofilm control (Supra) can reduce clinical and microbiological subgingival indicators in patients with moderate-to-severe chronic periodontitis, whether smokers or never-smokers. ${ }^{10,11}$ Parallel to these effects, a reduction in GCF volume was observed. ${ }^{12}$ However, whether the concentration of the inflammatory biomarkers is also influenced by the Supra regimen and to what extent this concentration is altered by tobacco exposure are unknown. To our knowledge, no comparisons have accounted for patient exposure to tobacco during a supragingival biofilm control regimen. The available literature focuses on subgingival scaling and root planing (SRP), delivered by different protocols and with conflicting results. ${ }^{13,14,15}$ However, current trends emphasize the importance of Supra control and the need to improve investigation in this field. . $10,11,12,16$

The aim of this secondary analysis was to investigate the effect of a 6-month supragingival control regimen on the subgingival concentration of IL-1 $\beta$ in smoker and never-smoker periodontitis patients. The hypothesis was that supragingival biofilm control reduces the concentration of IL-1 $\beta$, irrespective of tobacco exposure.

\section{Methodology}

\section{Study design, sample, and ethical considerations}

The present study is a secondary analysis of a single-arm clinical study..$^{10}$ Details of sample calculation, inclusion and exclusion criteria, patient compliance, and clinical findings have been previously described. ${ }^{10,12}$ Briefly, requiring a power of $80 \%$ to detect a difference of $0.80 \pm 1.0$ $\mathrm{mm}$ in periodontal probing depth (PPD), a sample size of 25 participants for each group (smokers and never-smokers) was calculated. Subjects were eligible if they had no systemic conditions that could affect the periodontium, no previous periodontal treatment, no use of anti-inflammatory drugs during the previous 3 months or antibiotics during the previous 6 months, and no chemical biofilm control prescriptions. Participants were included if they presented the following criteria: presence of at least 12 teeth, excluding third molars; clinical diagnosis of biofilm-induced gingivitis and moderate-to-severe generalized chronic periodontitis; at least four teeth with PPD $\leq 5 \mathrm{~mm}$ and four teeth with PPD $\geq 6 \mathrm{~mm}$, with supragingival biofilm, marginal bleeding, clinical attachment loss (CAL) and bleeding on probing (BOP).

Before the commencement of the original study, participants read and signed an informed-consent form. The study was in accordance with the Declaration of Helsinki and approved by the Research Ethics Committee of the Universidade Estadual Paulista-UNESP, Araraquara, Brazil (\#32/03). Subject protection was based on the clinical attachment level. During the study, subjects showing progression to $C A L \geq 2 \mathrm{~mm}$ were to be excluded. Fifty participants were included in the clinical study and six did not complete the study. ${ }^{10}$ No exclusions were due to CAL progression. ${ }^{10}$

For laboratory analysis, GCF samples from 43 participants were available: 23 never-smokers ( $47.3 \pm 6.7$ years, $41.7 \%$ males, $21.16 \pm 3.7$ teeth) and 20 smokers ( $45.8 \pm 5.1$ years, $55 \%$ males, $20.85 \pm 3.7$ teeth, $19.6 \pm 11.8$ cigarettes/day, $24.1 \pm 8.7$ years of smoking).

\section{Clinical examinations and GCF sampling}

Clinical examinations and GCF sampling were performed as previously described. ${ }^{10,12}$ GCF levels at 3 different time points (days 0,30, and 180) were analyzed.

A single examiner performed clinical examinations using a manual periodontal probe. Weighted $( \pm 1 \mathrm{~mm})$ kappa values for PPD were 0.98 and 0.95 before and during the study, respectively. Full-mouth periodontal examination (six sites per tooth: mesio-buccal, mid-buccal, disto-buccal, mesio-lingual, mid-lingual, and disto-lingual) was performed. PPD, in millimeters, and BOP, as a percentage of positive sites, were the clinical indicators used.

GCF samples were collected using Periopaper and assessed with a calibrated Periotron ${ }^{\circledR}$ (OraFlow, PlainView, New York, USA). GCF samples were harvested from four different sites drawn from 3-5 mm PPD sites and four from sites with 6-10 $\mathrm{mm}$ 
PPD per patient. Paperpoints were separately stored in sterilized tubes at a temperature of $-20^{\circ} \mathrm{C}$ until laboratory procedures were performed.

\section{Experimental intervention}

After baseline exams, patients were enrolled in a strict Supra regimen: calculus removal, extraction of nonrestorable teeth, endodontic treatment and temporary restoration, and oral hygiene instructions (OHI). The re-evaluation and reinforcement of $\mathrm{OHI}$ were performed on a weekly basis over 180 days based on visible plaque and gingival bleeding indices. Multi-tufted brushes, interdental devices, and fluoride toothpaste were provided as needed throughout the study. ${ }^{10}$ At the end of the study, patients received subgingival scaling and root planing.

\section{Laboratory procedures}

One calibrated examiner blinded to sample origin performed the laboratory procedures. Total amounts of IL-1 $\beta$ were assessed for each patient and each site on days 0,30 , and 180 using the enzyme-linked immunosorbent assay (ELISA). The sandwich ELISA technique was used with interleukin-1 $\beta$ EIA Kit (Cayman Chemical, Ann Arbor, USA).

Plates were prepared according to manufacturer instructions. A total of 1,032 samples were tested (in duplicate) in the three experimental periods. Assay sensitivity was $1.5 \mathrm{pg} / \mathrm{mL}$. Samples showing IL-1 $\beta$ concentrations below $1.5 \mathrm{pg} / \mathrm{mL}(\mathrm{n}=107)$ were considered as presenting the minimum concentration. Analyses were performed at the Cellular and Molecular Biology Laboratory at UNESP/Araraquara. The IL-1 $\beta$ concentration for each site was calculated by division of the total amount of IL-1 $\beta$ by the total volume of GCF.

\section{Statistical analysis}

The present analysis included data from sites where GCF was collected. The individual was considered the unit of analysis.

A robust varianceestimator (Huber/White/sandwich estimator of variance) was used to estimate standard errors (SE) adjusted for the correlation of data because of multiple sites within each subject. Comparisons of point estimates between never-smokers and smokers were performed using Wald tests. p-values were adjusted for multiple comparisons, and means \pm SE were calculated.

Because IL-1 $\beta$ concentration showed a non-normal distribution, a base 10-logarithm transformation was used to model IL-1 $\beta$ against multiple independent variables. Multivariate analysis using generalized estimating equations (GEE) with exchangeable correlation structure was then applied to evaluate the longitudinal effects of time, smoking status, PPD at the baseline, and BOP at 180 days on a log concentration of IL-1 $\beta$. Main-effects and interaction models are presented.

Analyses were performed using the Stata software (STATA 10 for Macintosh, Stata Corporation, College Station, USA). The significance level was set at $5 \%$.

\section{Results}

Table 1 shows baseline characteristics of the sample, considering only the sites subjected to GCF sampling. Overall (total sample) PPD value and IL-1 $\beta$ concentration were $5.0 \pm 0.1 \mathrm{~mm}$ and $3.2 \pm 0.3 \mathrm{pg} / \mu \mathrm{L}$, respectively. No significant differences were observed between smokers and never-smokers.

Figure 1 shows mean concentrations of IL- $1 \beta$ over time for never-smokers and smokers by experimental sites. Changes in IL-1 $\beta$ concentration followed a similar pattern in both groups, without significant intergroup differences: concentration significantly decreased from 3.2 to $1.9 \mathrm{pg} / \mathrm{ul}$.

Baseline concentrations of IL-1 $\beta$ were increased, with higher baseline PPD values in never-smokers and smokers (Figure 2). In both groups, IL-1 $\beta$ concentrations significantly decreased in all PPD categories from the baseline to 180 days. In

Table 1. Baseline data of the present sample considering only the experimental sites (mean \pm standard error).

\begin{tabular}{|c|c|c|c|c|}
\hline Variable & $\begin{array}{l}\text { Overall sample } \\
\quad(n=43)\end{array}$ & $\begin{array}{l}\text { Never-smokers } \\
\quad(\mathrm{n}=23)\end{array}$ & $\begin{array}{l}\text { Smokers } \\
(\mathrm{n}=20)\end{array}$ & $p^{a}$ \\
\hline PPD (mm) & $5.0 \pm 0.1$ & $4.9 \pm 0.2$ & $5.2 \pm 0.1$ & 0.13 \\
\hline BOP (\%) & $100 \pm 0$ & $100 \pm 0$ & $100 \pm 0$ & 1.00 \\
\hline $\mathrm{IL}-1 \beta(\mathrm{pg} / \mu \mathrm{L})$ & $3.2 \pm 0.3$ & $3.1 \pm 0.5$ & $3.3 \pm 0.4$ & 0.86 \\
\hline \multicolumn{5}{|c|}{$\begin{array}{l}\text { BOP: bleeding on probing; Experimental sites: sites submitted to } \\
\text { gingival crevicular fluid sampling; } p \text { : } p \text {-value; PPD: periodontal } \\
\text { probing depth. } \\
\text { a Comparison between smokers and never-smokers, Mann-Whitney } \\
U \text { test. }\end{array}$} \\
\hline
\end{tabular}


never-smokers, IL-1 $\beta$ slightly increased from the baseline to 30 days but leveled off at 180 days in sites with baseline PPD of 1-3 and $\geq 6 \mathrm{~mm}$. In contrast, no significant differences were observed between never-smokers and smokers in any PPD category over the experimental period.

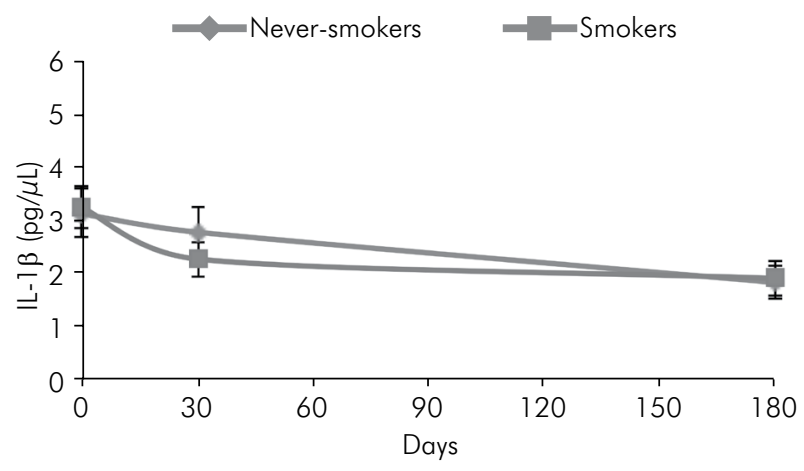

Figure 1. Means (with standard-error bars) of IL-1 $\beta$ concentration over the study period by smoking status and experimental site. There were no significant differences between never-smokers and smokers.

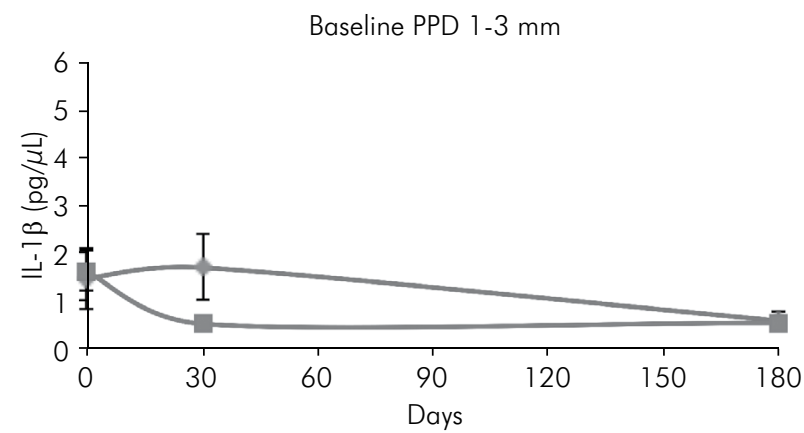

Table 2 shows the multivariate GEE model of the effects of time, smoking status, and baseline PPD and BOP at the 180-day examination on the $\log$ concentrations of IL- $1 \beta$. IL- $1 \beta$ concentration significantly decreased over the 180-day period. Smoking habit was not associated with changes in IL-1 $\beta$. Higher baseline PPD and the presence of BOP at 180 days were associated with higher levels of IL-1 $\beta$ over time.

A significant interaction was observed between smoking status and BOP at 180 days (Figure 3). The multivariate GEE model of the interaction between these two variables is shown in Table 3 . Time and baseline PPD remained significantly associated with log IL-1 $\beta$. Higher concentrations of IL-1 $\beta$ were observed in sites that showed BOP at 180 days in both never-smokers $(b=0.39 \pm 0.07)$ and smokers $(b=0.24 \pm 0.09)$ as compared to sites with the absence of BOP in never-smokers. This finding indicates that the role of $\mathrm{BOP}$ is stronger than that of the smoking status with respect to the concentration of IL-1 $\beta$.

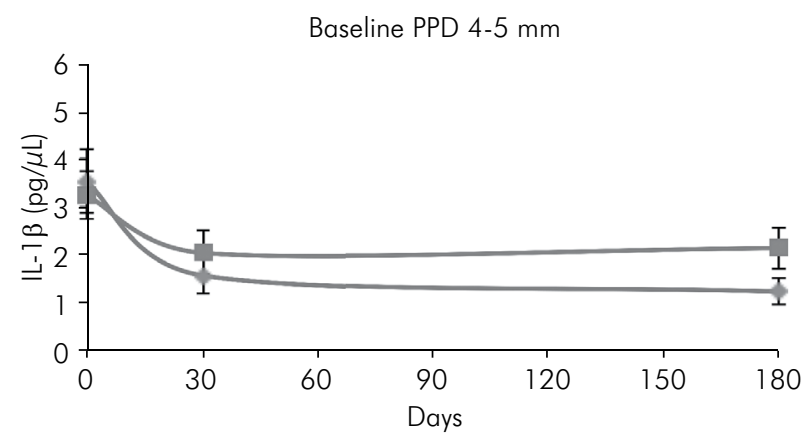

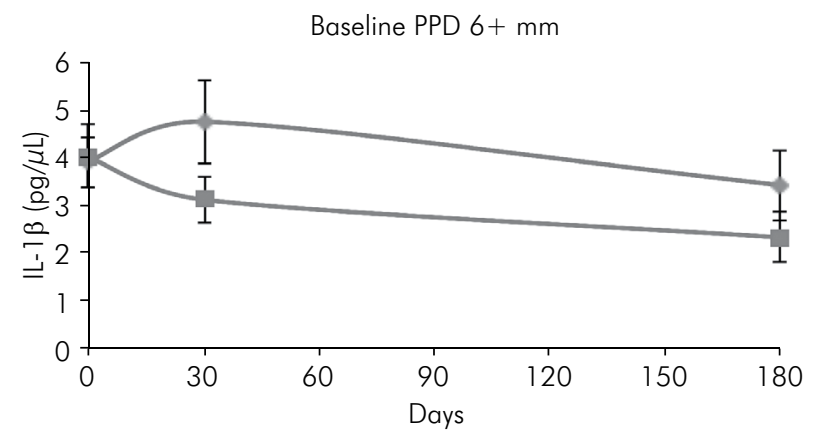

Figure 2. Means (with standard-error bars) of IL-1 $\beta$ concentraton over the study period by smoking status and baseline PPD values. There were no significant differences between never-smokers and smokers. 
Table 2. Multivariate GEE model of the effect of the time, smoking status, baseline PPD values and BOP at the 180-day examination on the log concentration of IL-1 $\beta$.

\begin{tabular}{lccc}
\hline \multirow{2}{*}{ Variable } & \multicolumn{3}{c}{ IL-1 $\beta$} \\
\cline { 2 - 4 } & Log $b$ & SE & $p$ \\
\hline Time & & & \\
Baseline & Ref. & & \\
30-days & -0.17 & 0.05 & 0.002 \\
180-days & -0.32 & 0.05 & $<0.001$ \\
Smoking status & & & \\
$\quad$ Never-smokers & Ref. & & \\
Smokers & -0.002 & 0.07 & 0.98 \\
Baseline PPD category & & & \\
1-3 mm & Ref. & & \\
4 -5 mm & 0.23 & 0.06 & $<0.001$ \\
$\quad \geq 6$ mm & 0.42 & 0.06 & $<0.001$ \\
BOP at 180 days & & & \\
$\quad$ Absent & Ref. & & \\
Present & 0.27 & 0.05 & $<0.001$ \\
\hline
\end{tabular}

BOP: bleeding on probing; GEE: generalized estimating equations; IL-1 $\beta$ : interleukin-1 $\beta$; $p$ : $p$-value; PPD: periodontal probing depth; SE: standard error.

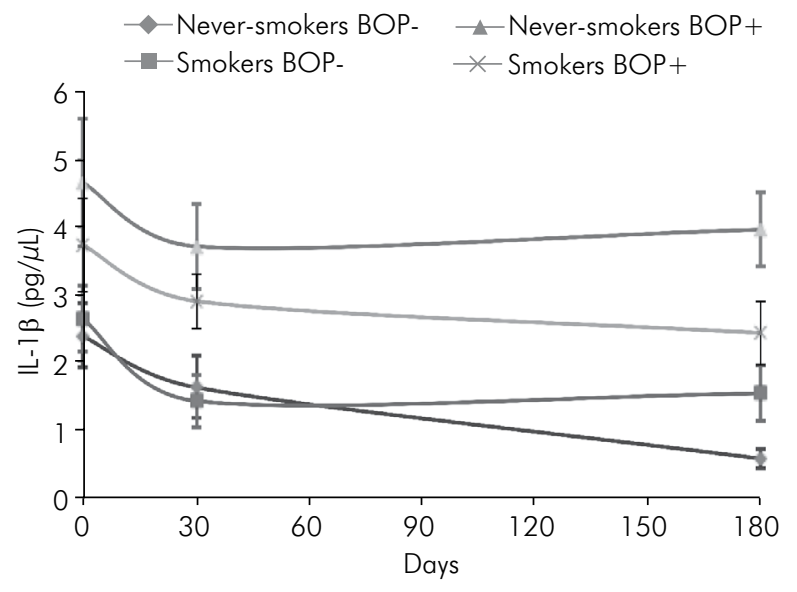

Figure 3. Means (with standard-error bars) of IL-1 $\beta$ concentration over the study period by smoking status and BOP at 180 days.

\section{Discussion}

The present study reports an immunological finding observed in a sample of patients previously assessed for clinical and microbiological parameters. ${ }^{10,11,12}$ This study extends the previous studies and contributes to the published literature with relevant data on the behavior of IL-1 $\beta$ following strict supragingival biofilm control in smoker and never-smoker periodontitis
Table 3. Multivariate GEE model of log concentration of $\mathrm{IL}-1 \beta$ including the interaction between BOP at 180 days and smoking status.

\begin{tabular}{|c|c|c|c|}
\hline \multirow{2}{*}{ Variable } & \multicolumn{3}{|c|}{ IL- $1 \beta$} \\
\hline & $\log b$ & SE & $\mathrm{p}$ \\
\hline \multicolumn{4}{|l|}{ Time } \\
\hline Baseline & Ref. & & \\
\hline 30-days & -0.17 & 0.05 & 0.002 \\
\hline 180-days & -0.32 & 0.05 & $<0.001$ \\
\hline \multicolumn{4}{|l|}{ Baseline PPD category } \\
\hline $1-3 \mathrm{~mm}$ & Ref. & & \\
\hline $4-5 \mathrm{~mm}$ & 0.25 & 0.06 & $<0.001$ \\
\hline$\geq 6 \mathrm{~mm}$ & 0.43 & 0.06 & $<0.001$ \\
\hline \multicolumn{4}{|c|}{ Interaction between 180-days BOP* smoking status } \\
\hline BOP-/never-smokers & Ref. & & \\
\hline BOP-/smokers & 0.09 & 0.08 & 0.263 \\
\hline $\mathrm{BOP}+/$ never-smokers & 0.39 & 0.07 & $<0.001$ \\
\hline $\mathrm{BOP}+/$ smokers & 0.24 & 0.09 & 0.006 \\
\hline
\end{tabular}

BOP-: absence of bleeding on probing; $\mathrm{BOP}+$ : presence of bleeding on probing; GEE: generalized estimating equations; IL-1 $\beta$ : interleukin-1 $\beta$; $\mathrm{p}$ : $\mathrm{p}$-value; PPD: periodontal probing depth; SE: standard error.

patients. Confirming the hypothesis, theSupra regimen led to a significant reduction in concentrations of IL-1 $\beta$, irrespective of smoking habit. Furthermore, baseline PPD values and persistent BOP were significantly associated with changes in IL-1 $\beta$ concentrations.

The present study is a secondary analysis from a clinical study, ${ }^{10}$ in which chronic periodontitis patients were maintained, over 6 months, solely with supragingival biofilm control. The high number of appointments delivered during the study deserves attention. The main clinical changes observed as early as the first 30 days of intervention ${ }^{10}$ show that this long period of supragingival control is time-consuming and not necessary to achieving desirable results from oral hygiene measures.

Among the immune-inflammatory biomarkers used to assess periodontal disease activity, IL-1 $\beta$ has been increasingly investigated. Some studies have reported an association between IL-1 $\beta$ concentration and clinical and microbiological parameters, ${ }^{17,18}$ and it has accordingly been used as a marker of the response to various periodontal therapies. ${ }^{19,20}$ However, despite its relevance to the assessment of host response, studies published to date have only focused on the effects of subgingival interventions 
on IL-1 $\beta$ concentration..$^{13,14,15}$ To our knowledge, the present study is the first to specifically focus on IL-1 $\beta$ concentration in GCF after a Supra regimen and to show a clear reduction in this biomarker over 180 days. This study is also the first to assess the impact of smoking habit, a well-known risk factor for periodontal disease, ${ }^{5,21}$ on IL-1 $\beta$ concentration during a Supra regimen. The findings of the present study more specifically suggest that IL-1 $\beta$ acts also as an important marker of subgingival response for the Supra regimen. Thus, our results shed some light on the etiopathogenic mechanisms regulating the subgingival-supragingival relationship. ${ }^{19}$ Still, the biomarker used here does not encompass all the subgingival responses that the periodontium may exhibit. On the basis of these results, it is reasonable to propose future studies in which more biomarkers could be included to produce a more complete scenario of the subgingival inflammatory/immunological response to this proposed model of Supra control in periodontitis patients.

Despite the adverse effects of smoking on periodontal tissues, in the present study both smokers and never-smokers showed significant reduction in IL-1 $\beta$ concentrations, with no intergroup differences. Nor were significant differences in supragingival status (visible plaque and marginal bleeding) observed between the groups over time. ${ }^{10}$ These findings underscore the importance of Supra in the management of periodontal infection, even when important risk factors are present. Comparisons of our results with the published literature are difficult due to the lack of other studies assessing the influence of smoking on patients subjected to supragingival intervention. Furthermore, there is still no agreement on the relationship between smoking habit and IL-1 $\beta$ concentration. Some authors have reported a positive effect, ${ }^{14,15}$ whereas others have reported a negative effect ${ }^{20,22,23}$ or no effect of smoking. ${ }^{21,24,25}$

An interaction between smoking/BOP and IL-1 $\beta$ concentration was observed on day 180 . This finding should be taken with care, as it suggests a role of the smoking habit. However, the finding that BOP- sites, in both smokers and never-smokers, showed significantly lower concentrations of IL-1 $\beta$ than $\mathrm{BOP}+$ sites in the same smoking categories shows that the presence of $\mathrm{BOP}$ exerts a stronger influence than the smoking habit, meaning that tobacco alone does not interfere with the concentration of IL-1 $\beta$. An association between increased IL- $1 \beta$ concentration and sites with persisting BOP was also observed. Subgingival bleeding is considered to act as a carrier, transporting inflammatory/immunological markers from connective tissue to the periodontal pocket. It is thus expected that as long as a site remains positive to BOP, no reductions will be observed in IL-1 $\beta$ concentration. The absence of reduction in inflammatory markers in the presence of BOP is in accordance with the literature. ${ }^{26,27}$ Such discussion has an important implication on clinical practice: namely that the presence of subgingival bleeding may be a more important and reliable indicator of periodontal disease activity after therapy than the historically more often used indicator, the PPD values. ${ }^{28}$

Sites with PPD $\geq 5 \mathrm{~mm}$ are considered to have a limited or even negative response to Supra or to nonsurgical therapies. ${ }^{29}$ In the present study, the Supra control reduced IL-1 $\beta$ concentrations in shallow, moderate, and deep sites. Higher concentrations of IL-1 $\beta$ on day 180 were associated with higher baseline PPD values. This finding is expected, given that deeper pockets at baseline remain deeper at the end of the experimental period. This result is consistently observed in studies examining the response to various periodontal treatments. ${ }^{10,13,18,29}$ Cross-sectional and interventional studies have reported that higher PPD values, particularly BOP positive, showed significant higher IL-1 $\beta$ concentrations. ${ }^{2,4,13,30}$

\section{Conclusions}

A strict Supra regimen exerted a significant effect on the periodontal immune-inflammatory response, as revealed by reduction in the IL-1 $\beta$ concentrations on GCF over 180 days. Tobacco alone did not influence this response. The results also suggested that higher baseline PPD values and persistent BOP could predict changes in IL-1 $\beta$ concentration after a strict Supra regimen. 


\section{References}

1. Gamonal J, Acevedo A, Bascones A, Jorge O, Silva A. Levels of interleukin- 1 beta, -8 , and -10 and RANTES in gingival crevicular fluid and cell populations in adult periodontitis patients and the effect of periodontal treatment. J Periodontol. 2000;71(10):1535-45. doi:10.1902/jop.2000.71.10.153

2. Holmlund A, Hanstrom L, Lerner UH. Bone resorbing activity and cytokine levels in gingival crevicular fluid before and after treatment of periodontal disease. J Clin Periodontol. 2004;31(6):475-82. doi:10.1111/j.1600-051X.2004.00504.x

3. Hughes FJ, Turner W, Belibasakis G, Martuscelli G. Effects of growth factors and cytokines on osteoblast differentiation. Periodontol 2000. 2006;41(1):48-72. doi:10.1111/j.1600-0757.2006.00161.x

4. Hou LT, Liu CM, Liu BY, Lin SJ, Liao CS, Rossomando EF. Interleukin-1beta, clinical parameters and matched cellular-histopathologic changes of biopsied gingival tissue from periodontitis patients. J Periodontal Res. 2003;38(3):247-54. doi:10.1034/j.1600-0765.2003.02601.x

5. Albandar JM. Global risk factors and risk indicators for periodontal diseases. Periodontol 2000. 2002;29(1):177-206. doi:10.1034/j.1600-0757.2002.290109.x

6. Lee J, Taneja V, Vassallo R. Cigarette smoking and inflammation: cellular and molecular mechanisms. J Dent Res. 2012;91(2):142-9. doi:10.1177/0022034511421200

7. Rivera-Hidalgo F. Smoking and periodontal disease. Periodontol 2000. 2003;32(1):50-8. doi:10.1046/j.0906-6713.2003.03205.x

8. Fiorini T, Musskopf ML, Oppermann RV, Susin C. Is there a positive effect of smoking cessation on periodontal health? A systematic review. J Periodontol. 2014;85(1):83-91. doi:10.1902/jop.2013.130047

9. Javed F, Al-Rasheed A, Almas K, Romanos GE, Al-Hezaimi K. Effect of cigarette smoking on the clinical outcomes of periodontal surgical procedures. Am J Med Sci. 2012;343(1):78-84. doi:10.1097/MAJ.0b013e318228283b

10. Gomes SC, Piccinin FB, Susin C, Oppermann RV, Marcantonio RA. Effect of supragingival plaque control in smokers and never-smokers: 6-month evaluation of patients with periodontitis. J Periodontol. 2007;78(8):1515-21. doi:10.1902/jop.2007.060462

11. Gomes SC, Nonnenmacher C, Susin C, Oppermann RV, Mutters R, Marcantonio RA. The effect of a supragingival plaque-control regimen on the subgingival microbiota in smokers and never-smokers: evaluation by real-time polymerase chain reaction. J Periodontol. 2008;79(12):2297-304. doi:10.1902/jop.2008.070558

12. Gomes SC, Piccinin FB, Oppermann RV, Susin C, Marcantonio RA. The effect of smoking on gingival crevicular fluid volume during the treatment of gingivitis. Acta Odontol Latinoam. 2009;22(3):201-6.

13. Goutoudi P, Diza E, Arvanitidou M. Effect of periodontal therapy on crevicular fluid interleukin-1beta and interleukin-10 levels in chronic periodontitis. J Dent. 2004;32(7):511-20. doi:10.1016/j.jdent.2004.04.003

14. Rawlinson A, Grummitt JM, Walsh TF, Ian Douglas CW. Interleukin 1 and receptor antagonist levels in gingival crevicular fluid in heavy smokers versus non-smokers. J Clin Periodontol. 2003;30(1):42-8. doi:10.1034/j.1600-051X.2003.300107.x

15. Tymkiw KD, Thunell DH, Johnson GK, Joly S, Burnell $\mathrm{KK}$, Cavanaugh JE, et al. Influence of smoking on gingival crevicular fluid cytokines in severe chronic periodontitis. J Clin Periodontol. 2011;38(3):219-28. doi:10.1111/j.1600-051X.2010.01684.x

16. Jenkins WM, Said SH, Radvar M, Kinane DF. Effect of subgingival scaling during supportive therapy. J Clin Periodontol. 2000;27(8):590-6. doi:10.1034/j.1600-051x.2000.027008590.x

17. Van der Velden U, Varoufaki A, Hutter JW, Xu L, Timmerman MF, Van Winkelhoff AJ, et al. Effect of smoking and periodontal treatment on the subgingival microflora. J Clin Periodontol. 2003;30(7):603-10. doi:10.1034/j.1600-051X.2003.00080.x

18. Wan $\mathrm{CP}$, Leung WK, Wong MC, Wong RM, Wan $\mathrm{P}$, Lo EC, et al. Effects of smoking on healing response to non-surgical periodontal therapy: a multilevel modelling analysis. J Clin Periodontol. 2009;36(3):229-39. doi:10.1111/j.1600-051X.2008.01371.x

19. Tezal M, Scannapieco FA, Wactawski-Wende J, Grossi SG, Genco RJ. Supragingival plaque may modify the effects of subgingival bacteria on attachment loss. J Periodontol. 2006;77(5):808-13. doi:10.1902/jop.2006.050332

20. Zhong Y, Slade GD, Beck JD, Offenbacher S. Gingival crevicular fluid interleukin-1beta, prostaglandin E2 and periodontal status in a community population. J Clin Periodontol. 2007;34(4):285-93. doi:10.1111/j.1600-051X.2007.01057.x

21. Kamma JJ, Giannopoulou C, Vasdekis VG, Mombelli A. Cytokine profile in gingival crevicular fluid of aggressive periodontitis: influence of smoking and stress. J Clin Periodontol. 2004;31(10):894-902. doi:10.1111/j.1600-051X.2004.00585.x

22. Boström L, Linder LE, Bergstrom, J. Clinical expression of TNF-alpha in smoking-associated periodontal disease. J Clin Periodontol. 1998;25(10):767-73. doi:10.1111/j.1600-051X.1998.tb02368.x

23. Boström L, Linder LE, Bergstrom J. Smoking and crevicular fluid levels of IL-6 and TNF-alpha in periodontal disease. J Clin Periodontol. 1999;26(6):352-7. doi:10.1034/j.1600-051X.1999.260604.x

24. Boström L, Linder LE, Bergstrom J. Smoking and GCF levels of IL-1ßeta and IL-1ra in periodontal disease. J Clin Periodontol. 2000;27(4):250-5. doi:10.1034/j.1600-051x.2000.027004250.x 
25. Erdemir EO, Duran I, Haliloglu S. Effects of smoking on clinical parameters and the gingival crevicular fluid levels of IL-6 and TNFalpha in patients with chronic periodontitis. J Clin Periodontol. 2004;31(2):99-104. doi:10.1111/j.0303-6979.2004.00454.x

26. Figueredo CM, Ribeiro MS, Fischer RG, Gustafsson A. Increased interleukin-1beta concentration in gingival crevicular fluid as a characteristic of periodontitis. J Periodontol. 1999;70(12):1457-63. doi:10.1902/jop.1999.70.12.1457

27. Gorska R, Gregorek H, Kowalski J, Laskus-Perendyk A, Syczewska M, Madalinski K. Relationship between clinical parameters and cytokine profiles in inflamed gingival tissue and serum samples from patients with chronic periodontitis. J Clin Periodontol. 2003;30(12):1046-52. doi:10.1046/j.0303-6979.2003.00425.x
28. Claffey N, Nylund K, Kiger R, Garrett S, Egelberg J. Diagnostic predictability of scores of plaque, bleeding, suppuration and probing depth for probing attachment loss. $31 / 2$ years of observation following initial periodontal therapy. J Clin Periodontol. 1990;17(2):108-14. doi:10.1111/j.1600-051X.1990.tb01071.x

29. Heitz-Mayfield LJ, Trombelli L, Heitz F, Needleman I, Moles D. A systematic review of the effect of surgical debridement vs non-surgical debridement for the treatment of chronic periodontitis. J Clin Periodontol. 2002;29(Suppl 3):92-102; discussion 160-2. doi:10.1034/j.1600-051X.29.s3.5.x

30. Engebretson SP, Grbic JT, Singer R, Lamster IB. GCF IL-1ßeta profiles in periodontal disease. J Clin Periodontol. 2002;29(1):48-53. doi:10.1034/j.1600-051x.2002.290108.x 